\title{
Índices de estereotipia verbal en textos escritos por personas con diagnóstico de esquizofrenia*
}

\author{
Stereotypic verbal indices in written texts by people diagnosed \\ with schizophrenia
}

Alicia Figueroa-Barra ${ }^{* *}$, Carolina Martínez Herrera ${ }^{* * *}$

\section{RESUMEN}

Este estudio analiza textos escritos por pacientes diagnosticados con esquizofrenia, en el marco de una tarea de escritura creativa. Se analizaron y cuantificaron las estructuras tanto léxicas como gramaticales con el fin de encontrar evidencia lingüística que permita visualizar el deterioro funcional en las capacidades de automonitoreo, fundamentales en la elaboración de un escrito. Para lograr este fin, se aplicó el índice Type and Token Ratio, se evaluó la longitud de las palabras y de las oraciones; y, finalmente, se realizó una parametrización morfológica para visualizar el repertorio léxico por categorías gramaticales. Los resultados obtenidos en los grupos de participantes con patología evidencian un desempeño deficiente en los indicadores estudiados. Esto demuestra que es posible proponer una caracterización operacionalizable de rasgos diferenciales en la escritura de personas con diagnóstico de esquizofrenia.
Palabras clave: escritura creativa, esquizofrenia, índices de estereotipia verbal.

El presente trabajo se enmarca en el Proyecto Fondecyt de Iniciación 11191122: "Análisis de marcadores lingüísticos de Esquizofrenia en población con estados mentales de alto riesgo de psicosis (EMARS)" cuya investigadora responsable es Alicia I. Figueroa-Barra.

** Chilena. Doctora en Filología Hispánica, Universidad de Valladolid. Profesora asistente Departamento de Psiquiatría y Salud Mental Sur de la Universidad de Chile. Investigadora asociada del Laboratorio de Psiquiatría Traslacional, Psiquislab, de la Universidad de Chile. Santiago, Chile. aliciafigueroa@uchile.cl

*** Chilena. Estudiante del Doctorado en Psicología, Pontificia Universidad Católica de Chile, MA in Linguistics, University College London. Académica de la Universidad de Valparaíso, investigadora doctoral del Laboratorio de Psiquiatría Traslacional, Psiquislab, de la Universidad de Chile. Santiago, Chile. carolina.martinez@uv.cl 


\begin{abstract}
This study analyzes written texts by patients diagnosed with schizophrenia within a creative writing task framework. Both lexical and grammatical structures were analyzed and quantified to find linguistic evidence, which allows the visualization of functional deterioration in self-monitoring abilities, fundamental in writing. To achieve this, we applied the Type and Token Ratio, and we evaluated the length of words and sentences; and, finally, we carried out a morphological parameterization to visualize the lexical repertoire by grammatical categories. The results obtained in the groups of participants with pathology show a poor performance in the indicators studied. This indicates that it is possible to propose an operable characterization of differential traits in the writing of people diagnosed with schizophrenia.
\end{abstract} Keywords: creative writing, schizophrenia, stereotypic verbal indices. 


\section{Introducción}

En el presente estudio abordamos los índices de estereotipia en textos escritos por personas con diagnóstico de esquizofrenia en una tarea de escritura creativa. Las estereotipias son repeticiones rituales no intencionales que pueden revelar una disminución en las capacidades de planificación y en las habilidades de procesamiento de la información. De modo general, las estereotipias verbales se consideran un comportamiento ritual, no propositivo y, por tanto, no comunicativo. En enfermedades neurodegenerativas como las afasias es frecuente el empleo de repeticiones de morfemas o ítems léxicos con función de relleno. En psicosis como la esquizofrenia es común que se repitan elementos discursivos que están descontextualizados respecto de la información central, o bien, que carecen de valor informativo, por ejemplo, las llamadas asociaciones consonánticas. En cuanto a su presencia en patologías infantiles, son visibles en niños del espectro autista o con retraso mental. Los trabajos experimentales sobre el lenguaje infantil de Miller (1981), Miller y Chapman (1981) y Fletcher (1985), aportaron evidencia acerca de la progresión consistente que poseen las capacidades léxicas desde la infancia, la cual es factible de ser parametrizada e incorporada en herramientas clínicas con potencial para diagnosticar discapacidades cognitivas.

Ahora bien, en cuanto a la presencia de estereotipias en tareas de escritura, aparecen como marcas lingüísticas ritualizadas, tales como frases y expresiones generalizadoras y muy recurridas, por lo que resultan poco ingeniosas y simplificadoras. La repetición de ítems léxicos o imágenes conceptuales basadas en lugares comunes se emplean sin fines comunicativos relevantes, aun cuando en ocasiones cumplen una función estética. No obstante, entre los textos escritos por periodistas profesionales no debe sorprendernos el empleo de estas marcas como complementos informativos. A causa de su versatilidad, pueden contribuir a configurar un estilo particular identificable del perfil editorial o del escritor en cuestión. Asimismo, también es posible identificar estas marcas entre escritores literarios profesionales, que en buenas cuentas, se valen de estas marcas como distinción estilística. En suma, la presencia de estereotipias se ajusta a una falta de experticia del escritor, o bien, a un giro estilístico calculado. 
En el presente trabajo hemos explorado textos escritos por personas con diagnóstico de esquizofrenia en una tarea creativa. Nuestro objetivo se centró, específicamente, en la exploración de las estructuras léxicas de dichos textos, con especial atención en estos recursos estereotípicos. Con este fin se analizó el volumen léxico, aplicando el índice Type and Token ratio, y se evaluaron los promedios de la longitud de las palabras y de la longitud de las oraciones. Junto con estas mediciones se realizó una parametrización morfológica para visualizar el repertorio léxico por categorías gramaticales. La disminución de estos indicadores se relaciona en forma directa con un deterioro funcional en las capacidades de automonitoreo, fundamentales en la elaboración de un escrito. Esto, debido a que de acuerdo con el modelo de Hayes y Flower (1980) inclusive aquellos escritores catalogados como inexpertos, realizan revisiones que permiten detectar errores como los analizados en este artículo, con el fin de evitarlos.

El objetivo principal de esta investigación fue comparar los recursos de la producción verbal en textos escritos entre personas con diagnóstico de esquizofrenia y en controles sanos. Para este propósito se utilizaron 20 textos correspondientes a una tarea de escritura, distribuidos en dos submuestras: 10 textos de personas con diagnóstico de esquizofrenia crónica, y de primer episodio, y 10 textos de personas en controles sanos. Todos los participantes del estudio son escritores inexpertos y ocasionales.

Cabe destacar que nos interesa explorar tareas de escritura con el fin de pesquisar evidencia lingüística concordante con el lenguaje desviado que se ha descrito como una característica definitoria de la esquizofrenia (Crow, 1997). Suscribimos a la idea de que, si el lenguaje no se desarrolla normalmente, el pensamiento también se ve alterado o empobrecido. Por lo tanto, la presencia de estereotipias verbales como recurso creativo entrega pistas acerca de las dificultades en la planificación y automonitoreo (Zimmerer et al., 2017). Basándonos en la perspectiva no cartesiana de Hinzen y Sheehan (2013), buscamos evidencia lingüística que nos aproxime a una mejor comprensión de la relación del lenguaje con formas específicas de pensamiento y referencialidad en hablantes con psicosis. 


\section{Antecedentes teóricos}

\section{La escritura}

Tal vez la necesidad de trascender a la evanescencia del habla es una de las razones que nos incita a escribir. En un amplio sentido, la tarea de escritura nos proporciona un espacio guiado por la reflexión para disponer de ideas e información trascendente (Olson, 1998). Desde una dimensión neurocognitiva, en la producción de mensajes escritos, se coordinan todas las estructuras cerebrales vinculadas con el lenguaje, los sistemas de memoria y el pensamiento (van Dijk y Kintsch, 1978). Por otra parte, la escritura requiere una práctica intensiva constante que demanda altos niveles de automonitoreo.

Se han propuesto, fundamentalmente, dos tipos de modelos teóricos para dar cuenta del proceso de escritura. Entre ellos, se distinguen los que señalan procesos cognitivos de bajo nivel o mecánicos, y los que proponen la participación de procesos de alto nivel o sustantivos (Ramón et al., 2018). El modelo de Hayes y Flower (1990) corresponde a este segundo tipo. Los autores consideran como unidades de análisis constituyentes del modelo de escritura los procesos cognitivos de alto nivel. Proponen que las distintas etapas de la escritura se pueden desarrollar mediante tres tareas metacognitivas:

- la planificación, que involucra la generación, jerarquización y organización de las ideas;

- redacción o textualizar considerando el registro adecuado, según el tema, la intención y el público destinatario; finalmente

- revisar o releer el escrito, con el fin de identificar y resolver problemas textuales.

Teniendo en cuenta estas necesidades, este modelo resulta de particular interés, pues plantea la importancia de desarrollar de manera consciente y activa el proceso de escritura, como puede apreciarse en la Figura 1. 


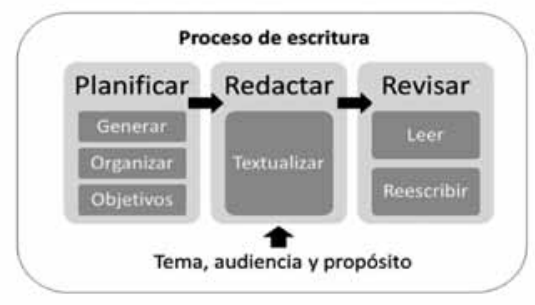

Figura 1. Proceso de escritura

Fuente: Elaboración propia sobre la base de Hayes y Flower (1990).

La importancia del proceso de elaboración de un texto es uno de los nudos críticos en los procesos de escritura. La escritura representa un medio de registro o comunicación y es, además, un instrumento que permite desarrollar, revisar, y transformar el propio saber (Carlino, 2009; Cassany, 2002; Ong, 1987). Una importante línea de investigación en psicolingüística corresponde al análisis del proceso de composición de la escritura. Por ejemplo, el interés de Cassany (2002) es determinar las diferentes fases de desarrollo que ocurren en el proceso de escritura y que abarcan desde el momento en que el escritor decide escribir hasta que termina una versión definitiva del texto.

A partir de estas investigaciones surgió el interés por comprender las estrategias que utilizan los escritores en la composición textual. Es así como se determinó la existencia de diferencias significativas entre quienes son categorizados como, escritores expertos, o como escritores inexpertos. Las distinciones entre ambas categorías se relacionan con la calidad de sus escritos. Por un lado, los escritores expertos recurren a subprocesos en la elaboración de la escritura organizados en etapas. Por ejemplo, en una primera etapa, realizan una búsqueda, organización y desarrollo de sus ideas; luego, en una segunda, redactan, evalúan y revisan su retórica. Por lo mismo, desarrollan mayor conciencia de su proceso de escritura y se adaptan al contexto de elaboración. En cambio, los escritores no expertos se limitan a capturar el flujo del pensamiento, en consecuencia, no generan ideas previas, ni las organizan, y solo se concentran en escribir sin releer, ni revisar su contenido o prosa.

Dentro de las estrategias de revisión del texto, los escritores más eficientes son aquellos que elaboran estructuras oracionales equili- 
bradas, escogen los ítems léxicos de forma asertiva y concordante con el tema y, además, evitan formulaciones decorativas que no aportan información pertinente. Por el contrario, el grupo de escritores inexpertos no planifica, no revisa, no reescribe, no establece propósitos, no adecúa el texto a la audiencia, organiza el texto de manera arbitraria y no desarrolla el contenido de acuerdo con los aspectos retóricos.

Teniendo en cuenta que el lenguaje es un sistema integrador de las funciones cognitivas, la elaboración de un texto implica la optimización de estrategias lingüístico-textuales, tales como la coherencia y cohesión, adecuación léxica, topicalización, y especialmente, la intencionalidad. En buenas cuentas, existe una ensambladura sutil que vertebra el mantenimiento del tema, los tópicos, y la distribución de la carga informativa en el texto (Figueroa et al., 2017). En cuanto a las estereotipias verbales en la escritura, estas funcionan como evidencia de un automonitoreo escaso o superficial. Como señala Cassany (2002), la reflexión acerca del proceso de elaboración de un texto es una tarea metacognitiva que demanda entrenamiento, por ello, es posible que los escritores inexpertos presenten mayor cantidad de estructuras estereotipadas.

\section{Procesos cerebrales de la escritura}

La escritura demanda un alto grado de coordinación interhemisférica. Asimismo, se produce una integración sensoriomotora en los dominios visoespaciales. Estudios como el de Potgieser et al. (2015) probaron, mediante un paradigma de $\mathrm{fMRI}^{1}$, la hipótesis de que los componentes espaciales y del lenguaje se encuentran en circuitos lateralizados, pero altamente coordinados entre sí. Esto tendría implicaciones directas en las demandas en tareas de escritura.

Los procesos cerebrales que involucran la planificación y ejecución de la escritura son operados por circuitos neuronales, cuya función es organizar la información, y facilitar el acceso a los datos almacenados en los sistemas de memoria. Este es el caso del proceso de activación del Lexicón, que es una estructura interna en la que almacenamos los significados de las palabras conocidas. Cuando se activa este almacén,

1 Functional magnetic resonance imaging (imagen por resonancia magnética funcional). 
podemos escoger opciones léxicas de mayor pertinencia, según el contexto de escritura (Cuetos, 2012).

El lóbulo frontal es otra área cerebral que juega un papel importante en los procesos cognitivos superiores. Permite realizar funciones de abstracción y razonamiento, además, se correlaciona con los sistemas de memoria verbal, abstracción, atención y velocidad psicomotriz, contribuyendo al proceso de planificación de la escritura (Altemeier et al., 2006; Baddeley, 2003). En cuanto al rol del lóbulo temporal, está el de facilitar la discriminación gráfica y fonológica, permitiendo la ejecución física de la escritura. Respecto de la motricidad de la escritura, es el lóbulo parietal el responsable de la coordinación óculo-manual. Se realiza una representación de la forma de las palabras, su ortografía y su ejecución mediante la coordinación ojo-mano. Berninger y Winn (2006) señalan la especial importancia de tres áreas relacionadas con los procesos de escritura. Son áreas cerebrales que se suman a la activación de redes neuronales específicas, según la tarea en ejecución: el área de Exner, el lóbulo parietal superior, y la región premotora frontal del lóbulo frontal izquierdo (Cuetos, 2012), como puede verse representado en la Figura 2.

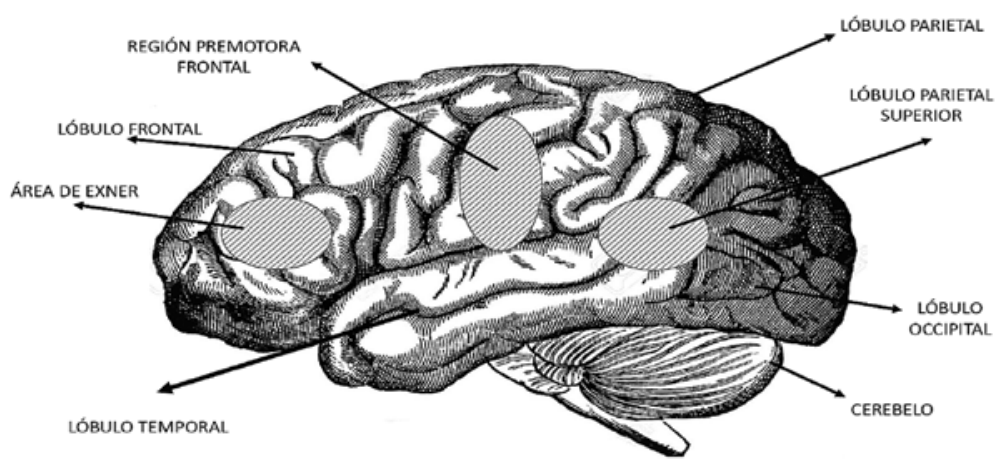

Figura 2. Áreas del cerebro involucradas en el proceso de escritura Fuente: Elaboración propia, adaptado de 32549865 @ Anthony Baggett | Dreamstime. com

En suma, la tarea de elaborar un escrito es una de las funciones lingüísticas de mayor complejidad cognitiva, además, implica la coordinación cerebral exitosa de diversas áreas corticales. El escritor se ve sometido a un trabajo intencional que requiere de un sofisticado proceso 
de automonitorización, con el fin de establecer relaciones lógicas entre las diferentes estructuras léxicas, semánticas y gramaticales (Liederman et al., 1983). El deterioro de porciones del lóbulo temporal y frontal se asocia con la disminución de la funcionalidad en la planificación lingüística en esquizofrenia, (Benson y Stuss, 1990; Okocha y Toone, 1992). A su vez, ambos circuitos corticales, frontales y temporales, han sido implicados en modelos recientes para explicar aspectos de la neuropatología de la esquizofrenia (Datta y Arnsten, 2018; Pittman-Polletta et al., 2015). Los desórdenes, las alteraciones, o las perturbaciones en el lenguaje en la esquizofrenia se manifiestan como características que se apartan de los parámetros lingüísticos normales, representan auténticos marcadores de rasgo inherentes a la patología (Figueroa Barra, 2015; Nuechterlein y Dawson, 1984). Los llamados marcadores de rasgo son las características psicopatológicas incipientes que permiten la elaboración de modelos de predicción de las enfermedades mentales.

\section{La escritura en la esquizofrenia}

La esquizofrenia es una enfermedad mental grave de carácter psicótico con síntomas positivos y negativos que provoca alteraciones emocionales, conductuales, sensoriales, psicomotoras y cognitivas (Andreasen y Grove, 1986; Castilla del Pino y Ruíz Vargas, 1991; Crow, 1997; McKenna y Oh, 2005). Esta patología provoca invalidez y deterioro, pues surge en los estadios tempranos del ciclo vital impidiendo el desarrollo, la integración y la autovalencia de los afectados. Su incidencia entre la población mundial es del $1 \%$, sin distinción de sexo, raza o clase social. La esquizofrenia produce una serie de cambios en los circuitos cerebrales responsables de la sincronía de procesos interhemisféricos, lo que afecta en forma directa el desempeño en las tareas de escritura. Asimismo, desde sus etapas iniciales puede aparecer un deterioro cognitivo que afecta otras áreas relacionadas con la atención, la memoria o las funciones ejecutivas.

Dentro del cuadro patológico general es frecuente encontrar TFP (trastorno formal del pensamiento), que consiste en un grupo de síntomas que provocan desorganización del pensamiento. Su evaluación se realiza sobre la base de la desestructuración del lenguaje y sus componentes. La evaluación del TFP ha sido propuesta por psiquiatras, entre los que destaca el trabajo de Andreasen y Grove (1986), y poste- 
riormente por Kircher et al. (2014). También se evalúa la desorganización del pensamiento con indicadores clínicos presentes en escalas e instrumentos estandarizados como PANSS, MOCA, GAF² y otros. En el caso de los instrumentos que miden TFP en psiquiatría, es necesario precisar que no aplican criterios lingüísticos específicos, ni tampoco son aplicados por lingüistas, lo cual impide que sea posible identificar manifestaciones lingüísticas útiles para diferenciar entre síntomas diversos. Para este estudio incluimos solo un $30 \%$ de escritores con diagnóstico de esquizofrenia que presentaban TFP.

Por otra parte, las tareas de escritura proporcionan una importante instancia para ejercitar la creatividad, de modo que son frecuentes entre las acciones terapéuticas aplicadas en esquizofrenia. Junto con la musicoterapia y la arteterapia, las sesiones de trabajo en escritura libre aportan enfoques para abordar algún aspecto específico de la experiencia mórbida, además, contribuyen a mejorar la autoestima y la confianza de los usuarios de programas de salud mental. Existen diferentes modalidades de trabajo terapéutico de la escritura, muchas de las cuales se convierten en trabajos literarios colectivos, cuya publicación es una valiosa forma de visibilizar las habilidades expresivas de las personas afectadas por esta psicosis. En cuanto a los manuscritos empleados en este trabajo, corresponden a esta modalidad de escritura libre en el marco de la terapia.

\section{Matriz analítica}

En este estudio tomaremos en cuenta los siguientes indicadores de estereotipias verbales en textos elaborados por personas con diagnóstico de esquizofrenia, en el marco de una tarea creativa: Type and Token Ratio, longitud de las palabras, longitud de las oraciones y categorías gramaticales.

\section{Type and Token Ratio (TTR)}

Este índice permite medir la variedad o la pobreza del léxico del hablante o escritor, basándose en el cálculo de la relación entre el número de palabras diferentes sobre el número total de palabras de un texto

2 PANSS: Positive and Negative Syndrome Scale; MOCA: Montreal Cognitive Assessment; GAF: Global Assessment of Functioning. 
escrito o hablado (Kirk, 1951; Malvern et al., 2004; Retherford y Choe, 1993). Cuanto más alto es el resultado, mayor variedad o riqueza de vocabulario manifiesta el texto (Bardin, 1991). El rango de valores va de 0 a 1 , entonces, si los valores son cercanos a 0 , ello sería indicador de una repetición de ítems léxicos; por el contrario, si los valores son cercanos a 1, sería indicativo de una menor repetición y, por tanto, de una mayor diversidad léxica.

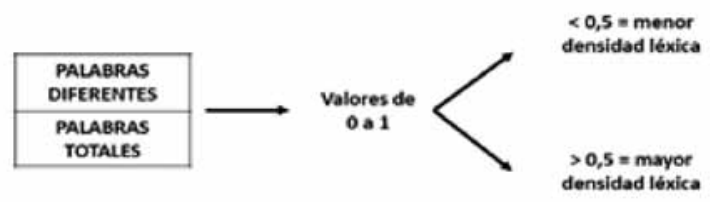

Figura 3. Type and Token Ratio

Fuente: Elaboración propia.

\section{Longitud de las palabras}

Este índice se calcula por el número de sílabas por palabra, considerando que la composición silábica y frecuencia de aparición en español se concentra en palabras bisilábicas, es decir, compuestas por dos sílabas (Escandell, 2014; RAE, s.f ${ }^{3}$ ). Este indicador surge de la conocida ley de Zipf (1972), que señala la presencia de un patrón estadístico en un texto. El uso de las palabras más frecuentes está expresado por valores constantes, es decir que el ítem léxico $\mathrm{X}$ tiene $(f)$ probabilidades de ocurrencia en un texto. Esta ley también permite discriminar la amplitud del vocabulario empleado, dada por la dependencia de la frecuencia de aparición respecto del número de palabras usadas en un texto, y que en español serían palabras de dos o tres sílabas. Mientras más reducido el vocabulario, mayor será la frecuencia de aparición, lo que se verá reflejado en una mayor o menor complejidad léxica.

\section{Longitud de las oraciones}

Esta medida se obtiene por el cálculo del número medio de palabras por oración. En cuanto a la lectura de un texto, es evidente que la di-

3 http://web.frl.es/CREA/view/inicioExterno.view;jsessionid=3DA47A6802E0F0094 E4B28428C9422FA 
ficultad en la comprensión también se relaciona con la longitud de las oraciones, y con su complejidad estructural. Se ha observado que en los textos de difusión, como por ejemplo revistas o infografías, se emplean de preferencia oraciones breves para facilitar la lectura y la comprensión. Por el contrario, en textos teóricos o especializados, es frecuente que las oraciones sean de mayor longitud, lo que demanda un mayor esfuerzo comprensivo por parte del lector.

Los textos escritos por personas con esquizofrenia pueden presentar problemas de cohesión relevantes, especialmente, cuando elaboran frases de una longitud extensa. Addington y Addington (2000) descubrieron que, al superar tres o cuatro elementos verbales, la cohesión empeora drásticamente entre personas con esquizofrenia. De esta manera, también podemos señalar que, a mayor cantidad de elementos oracionales, habrá una mayor laxitud referencial y una menor densidad cohesiva (Figueroa, en prensa). Ahora bien, hemos observado que la preferencia por el empleo de oraciones simples y breves también reduce la complejidad sintáctica. Morice y Ingram (1983) demostraron que esta reducción de la complejidad sería la causa de la llamada pobreza del habla, uno de los indicadores de TFP.

\section{Categorías gramaticales}

El análisis morfológico de los textos permite extraer información relacionada con las categorías gramaticales de las palabras, y su flexión morfológica. Esta medida la incluimos para comparar el contingente de recursos léxicos entre los grupos, y verificar si existían diferencias en cuanto al volumen por categoría gramatical empleado. No realizamos un análisis de desambiguación gramatical, puesto que no se incluye en los objetivos de este estudio en particular. Las categorías que se incluyeron son verbos, sustantivos, adjetivos, adverbios, pronombres, preposiciones, artículos y conjunciones.

\section{Material y método}

\section{La muestra}

El presente estudio incluyó una muestra de 20 textos distribuidos como sigue: 10 textos escritos por personas con diagnóstico de esquizofrenia, y 10 textos escritos por personas sin patología psiquiátrica, reclutadas para este estudio. Los textos de esta investigación fueron escritos en el 
marco del trabajo terapéutico regular en el caso del grupo con esquizofrenia, y de una actividad creativa en el caso del grupo control. En esta actividad terapéutica de escritura se unificaron los textos para la obtención de materiales adecuados para el estudio. Al tratarse de una tarea creativa fue necesario descartar textos que no constituían narraciones tales como ideas sueltas, pequeños poemas, o bocetos de escritura. Además, esta actividad terapéutica era de carácter voluntario y circunscrita a otro tipo de actividades propias del trabajo de un hospital de día ${ }^{4}$, por ello, nuestra muestra resultó acotada. En una experiencia piloto previa, esto es, el programa CONECTA-2 (Figueroa, et al., 2019) probamos la utilidad de la escritura narrativa como tarea expresiva y terapéutica, por ello, la aplicación de la tarea de escritura de este estudio fue realizada por la misma investigadora participante en dicho programa.

La estructura de los textos seleccionados es narrativa y se trata de cartas e historias escritas con lápiz y papel. Tienen una longitud promedio de 350 palabras, límite que hemos considerado en este trabajo, para homogeneizar la muestra, reuniendo un total aproximado de 7.000 palabras. Esta tarea fue ejecutada con un amplio margen de tiempo que incluyó dos sesiones completas de 90 minutos. El grupo de escritores con diagnóstico de esquizofrenia se divide en dos subgrupos: crónicos y de primer episodio (G1-CRE y G2-PEE). Los diagnósticos fueron confirmados por un equipo de psiquiatras, quienes utilizaron la estructura de la entrevista clínica para el DSM-IV -TR, SCID-P 5 (First et al. 2002) y la escala de síntomas positivos y negativos PANSS (Kay, et al., 1991). Todos los pacientes incluidos en este estudio estaban estabilizados psicopatológicamente, recibiendo dosis orales o de depósito de antipsicóticos. Del grupo de pacientes crónicos, tres de ellos presentaban TFP, no obstante, se encontraban compensados al momento de la aplicación de la tarea. El resto de los participantes con diagnóstico de esquizofrenia presentaban bajas puntuaciones en los indicadores que miden este trastorno.

4 Un hospital de día es un sistema de hospitalización parcial. Entre sus funciones está la de facilitar la interacción social del paciente disminuyendo su aislamiento, mejorar el cumplimiento del tratamiento suministrando la medicación, permitir tratamientos grupales y terapias ocupacionales, etc. El primer hospital de día fue creado en 1946 por D. Ewen Cameron (1901-1967) en Montreal (Canadá). https://psiquiatria.com/glosario/ index.php?wurl=hospital-de-dia

$5 \quad$ Structured Clinical Interview for DSM IV-I/P, SCID-P, (First et al. 2002) 
El grupo de control (G3-CT) corresponde a 10 participantes sin patología psiquiátrica, pareados con las características sociodemográficas de los sujetos con diagnóstico de esquizofrenia. Los controles elaboraron textos narrativos en el marco de una actividad de escritura creativa organizada para la comunidad, por uno de los centros de rehabilitación al que pertenecían los participantes de G2-PEE. De una muestra inicial de 25 textos, se escogieron 10 cuya estructura era equivalente a los producidos en las sesiones terapéuticas, y fue desarrollado en una sola sesión de 90 minutos. La participación para este estudio contó con un consentimiento informado escrito, firmado por los pacientes y un familiar o cuidador responsable; este consentimiento fue aprobado por el comité de ética científica de la Facultad de Medicina de la Universidad de Chile. En la Tabla 1 reseñamos las características de todos los participantes:

Tabla 1

Características de los participantes del estudio

\begin{tabular}{|c|c|c|c|c|c|c|}
\hline & G1-CRE & G2-PEE & G3-CT & PANSS & & \\
\hline $\mathrm{N}$ & 5 & 5 & 10 & TFP & 3 & - \\
\hline $\begin{array}{l}\text { Sexo, masc./ } \\
\text { fem. }\end{array}$ & $4 / 1$ & $1 / 4$ & $5 / 5$ & PANSS-P & $37,2(33,6)$ & 24,2 \\
\hline Edad & 43 & 20,4 & 26,7 & PANSS-N & $24,6(33,3)$ & 34,3 \\
\hline $\begin{array}{l}\text { Años } \\
\text { escolarización }\end{array}$ & 14 & 13 & 14 & PANSS -PG & $68,56(70,2)$ & 52,32 \\
\hline $\begin{array}{l}\text { Actividad } \\
\text { remunerada }\end{array}$ & 0,2 & 0,4 & 0,4 & $\begin{array}{l}\text { PANSS } \\
\text { Total }\end{array}$ & $111,96(132,11)$ & 110,82 \\
\hline $\begin{array}{l}\text { Duración } \\
\text { enfermedad }\end{array}$ & 14,6 & 1,4 & & & & \\
\hline
\end{tabular}

Fuente: Elaboración propia.

\section{Procedimiento de análisis}

- Para realizar el análisis de los índices de estereotipia se realizaron los siguientes procedimientos:

- Para calcular el TTR se empleó el parametrizador morfológico PARAMTEXT TIP ${ }^{6}$ (Carreras-Riudavets et al., 2011). De esta forma se obtuvo el dato de las palabras totales y palabras diferentes (sin palabras vacías) de los textos analizados.

6 https://tulengua.es/paramtext/ 
- Para medir la longitud de las palabras, calculamos el número de sílabas por palabra con el sitio separarensílabas.com (2018).

- La longitud de las oraciones fue calculada con separarensílabas. com (2018). Con esta información se calculó el promedio de palabras por oración.

- Las categorías gramaticales se clasificaron con PARAMTEXT TIP, que entrega información detallada acerca de la frecuencia de uso, las categorías morfológicas y léxicas, independientemente de su función gramatical en los textos.

\section{Procedimientos estadísticos}

El diseño de este estudio es transversal, comparativo y no experimental. Se realizó un análisis de las estructuras textuales que componen los escritos, a partir del cual se generaron las variables categorizadas. Al tratarse de un estudio con variables cuantitativas múltiples, utilizamos una prueba paramétrica ANOVA para el análisis de la varianza, para lo cual comprobamos el cumplimiento de la distribución normal de los datos conseguidos y homogeneidad de las varianzas. En todos estos casos, el grado de significación estadística se definió en $(p<, 05)$. El paquete estadístico que empleamos es el SPSS, versión 21 para Windows.

\section{Resultados}

A continuación se presentan los resultados por índices de estereotipia.

\section{TTR y estadio de la enfermedad}

Esta medida indica variedad o riqueza léxica en un texto. El análisis de los datos obtenidos revela una evidente diferencia entre los tres grupos de informantes. Las medidas de densidad léxica del G3-CT indican que el índice corresponde a [0,73], es decir, muy cercano a 1 ; de modo que corresponde a una mayor densidad léxica que en el grupo de CRE, que obtuvo $[0,6]$. Por el contrario, el índice de densidad léxica en el grupo de PEE alcanza [0,43], el índice de menor valor entre los tres grupos. Como se aprecia en el Figura 3, el valor de ANOVA es significativo estadísticamente $(F(2,17)=21,83, p<, 001)$. 


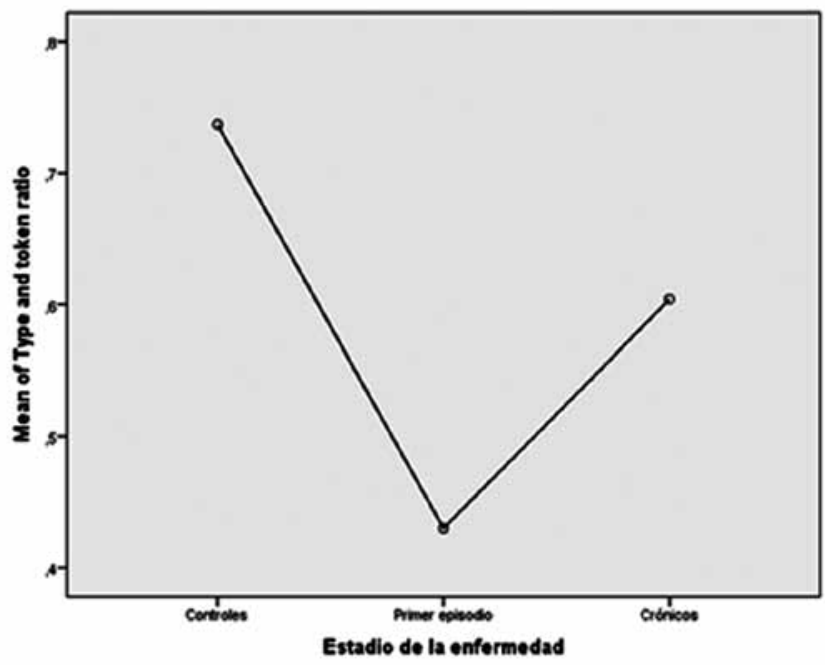

Figura 3. TTR estadio de la enfermedad Fuente: Elaboración propia.

A pesar de que la actividad de escritura contó con condiciones óptimas para la concentración, como tiempo y un espacio de trabajo confortable, se aprecia una diferencia significativa en la variedad léxica a favor de los controles (G3-CT), y en desmedro de los resultados obtenidos en los dos grupos de esquizofrenia (G1-CRE y G2-PEE). Estos hallazgos son concordantes con los estudios sobre léxico, en los cuales se describe un aumento en los tiempos de reacción, en tareas de decisión léxica. Asimismo, la investigación cuantitativa respecto del léxico ha demostrado una reducción en el volumen de palabras utilizado en tareas de escritura y de evocación (Allen et al., 1993). Esto nos hace pensar en que, posiblemente, una dificultad de acceso al léxico es uno de los obstáculos de mayor relevancia para una variedad léxica amplia. En este sentido, se observa que el uso de recursos léxicos con una intención estilística coherente es deficiente en los textos de los grupos G1-CRE y G2-PEE. Encontramos una proporción de palabras inusuales o rebuscadas que se han descrito como típicas del léxico en esquizofrenia, sumado al uso frecuente de palabras repetidas dentro del mismo texto. En la Figura 4 se muestra una comparación que ilustra la diferencia en este indicador entre los tres grupos: 


\section{TTR intergrupos}

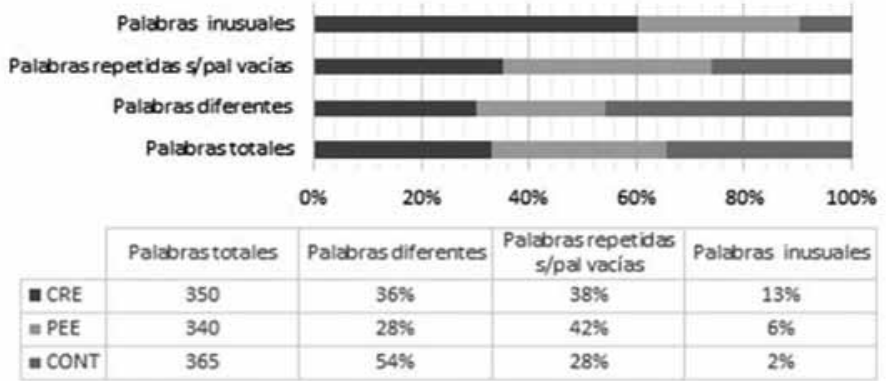

Figura 4. Palabras repetidas, palabras diferentes, total de palabras y palabras inusuales

Fuente: Elaboración propia.

\section{Longitud de las palabras}

Esta medida ilustra la mayor o menor complejidad léxica del hablante, a partir del conteo del número de sílabas por palabra. Se observa que el grupo control supera las dos sílabas por palabra, mientras que los CRE y PEE registran 1,9 y 1,7 respectivamente. Como se aprecia en la Figura 5 , el valor de ANOVA es $(F(2,17)=9,91, p<.001)$ significativo estadísticamente.

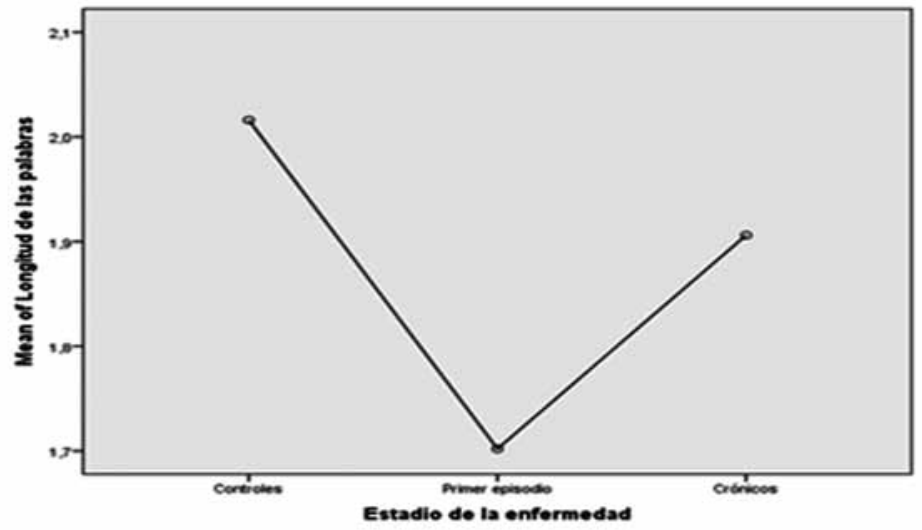

Figura 5. Longitud de las palabras

Fuente: Elaboración propia. 
No solo las diferencias estadísticas llaman la atención entre los grupos de escritores, ya que es posible inferir que estos resultados también se deben a un uso menos consciente de los recursos léxicos, y a una menor capacidad metacognitiva para escoger palabras pertinentes. Las posibles palabras clave se sobreutilizan en el texto, generando la impresión de recursos léxicos restringidos. Esta constante reutilización impide una progresión textual, generando la impresión de redundancia. Observamos que hay pocas o ninguna marca lingüística estratégica, y que el empleo de los ítems léxicos adopta fundamentalmente una función informativa, pese a que se trataba de una tarea creativa. En esquizofrenia, uno de los indicadores asociado al volumen léxico restringido es la llamada pobreza del habla, descrita por Andreasen y Grove (1986). Desde un punto de vista lingüístico podríamos describir la pobreza del habla como el uso de palabras genéricas como algo, cosa, cuestión, asunto, etc.; y también la preferencia por palabras de uso coloquial. Con ello, encontramos que el gran porcentaje del volumen léxico correspondía a palabras con una extensión aproximada de dos sílabas. Sumado a lo anterior, a pesar de este repertorio restringido, también se presentaron ítems léxicos rebuscados e inusuales que, sin embargo, no aportaron suficiente variedad léxica para equilibrar los textos.

\section{Longitud de las oraciones}

Esta medida se obtuvo por el cálculo del número medio de palabras por oración. Los datos resultantes de este análisis evidencian diferencias entre los tres grupos de informantes. En el caso del grupo control se observa una cantidad de 37,4 palabras por oración, mientras que el grupo G1-CRE registra 23,5 y el grupo G2-PEE 13,1 de palabras por oración. Como se aprecia en la Figura 6, el valor de ANOVA es $(F(2,17)$ $=21,12, p<, 001)$ significativo estadísticamente. 


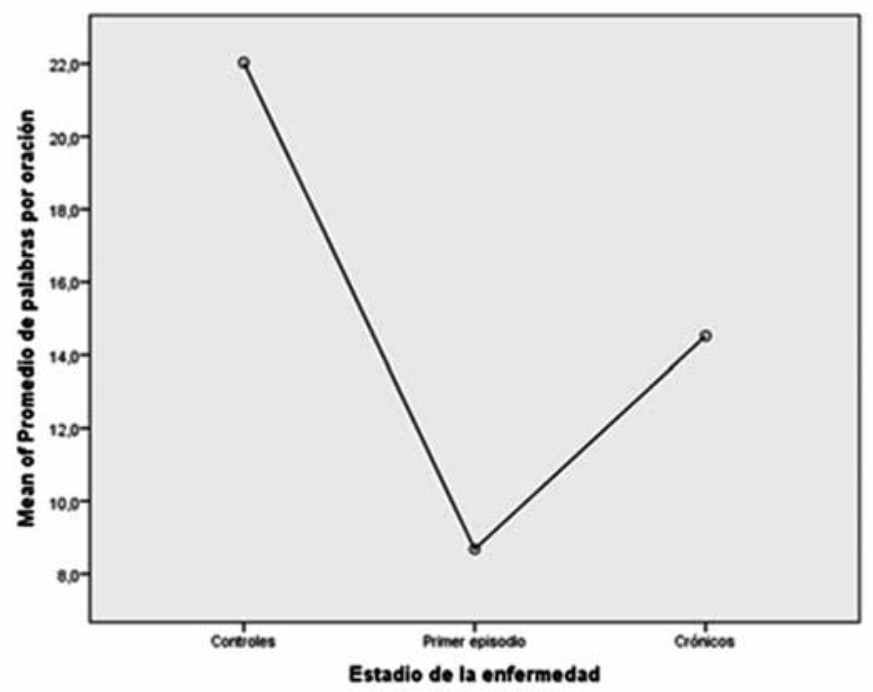

Figura 6. Longitud de las oraciones

Fuente: Elaboración propia.

Según la literatura científica, los textos presentan rasgos estilísticos y de diferentes niveles de complejidad, sobre la base de la longitud oracional promedio. La mayor distancia entre sujeto y verbo hace más susceptible la ocurrencia de anacolutos o estructuras erróneas gramaticalmente. Sin embargo, la brevedad de las oraciones genera una simplificación excesiva de las ideas. Dentro de nuestros hallazgos pudimos comprobar algunas ideas de Addington y Addington (2000) acerca de la aparición de problemas de cohesión en oraciones más extensas, en especial en el grupo de esquizofrenia crónica. En la comparación entre años de estudio, G1- CRE alcanzaron a estudiar más que G2- PEE (en este corpus), de modo que es probable que haya un factor etario y educacional que podría explicar esta diferencia.

\section{Categorías gramaticales}

Los resultados de la clasificación morfoléxica aportan luz acerca de los recursos presentes en los textos. Esta evaluación se tuvo en cuenta para comparar el uso y variedad de las categorías gramaticales en los textos, pero sin considerar la función gramatical específica. En la Tabla 2 se muestra el promedio de recursos léxicos entre los grupos de esqui- 
zofrenia y el grupo control. Además, en la Figura 7 se puede observar que, a pesar de que todas las categorías se encuentran representadas, el volumen por categoría es consistentemente más restringido en esquizofrenia.

Tabla 2

Promedio de recursos léxicos observables en los textos.

\begin{tabular}{lcccc}
\hline & CRE & PEE & CT & DE \\
\hline Verbos & 83,2 & 87,3 & 94,1 & 5,5 \\
\hline Sustantivos & 146,4 & 161,2 & 172 & 12,8 \\
\hline Adjetivos & 68,6 & 76,1 & 83,3 & 7,3 \\
\hline Adverbios & 35 & 36,1 & 37 & 1,0 \\
\hline Pronombres & 58,8 & 59,5 & 66,4 & 4,2 \\
\hline Preposiciones & 46,8 & 53,5 & 60,3 & 6,7 \\
\hline Artículos & 30,2 & 41,1 & 45,7 & 7,9 \\
\hline Conjunciones & 43,8 & 41,3 & 43 & 1,2 \\
\hline
\end{tabular}

Fuente: Elaboración propia.

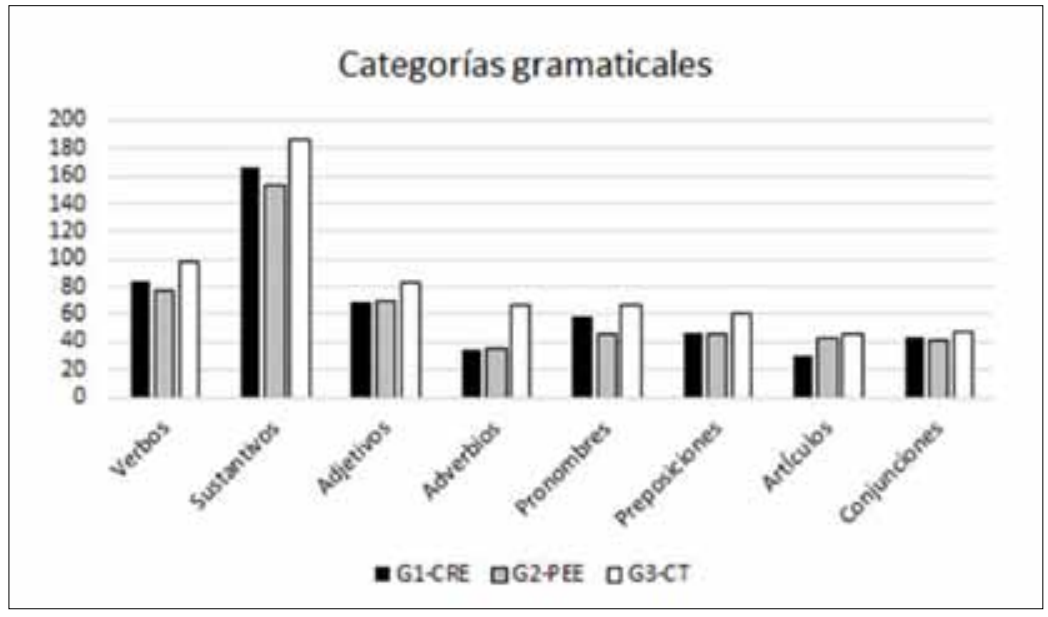

Figura 7. Categorías gramaticales Fuente: Elaboración propia.

\section{Discusión}

Si bien este estudio corresponde a una primera aproximación a una caracterización de recursos léxicos en tareas de escritura en esqui- 
zofrenia, podemos adelantar algunas interpretaciones iniciales. La primera de ellas es que las estereotipias verbales en esquizofrenia se manifiestan en forma perseverante, acompañan tanto las intervenciones orales espontáneas como el desempeño en tareas que requieren planificación y reflexión como la escritura. Por otra parte, a pesar de que parecen aumentar con el curso prolongado de la enfermedad, son observables en el primer episodio, aunque en esta fase impactan en específico el volumen y variabilidad del léxico.

\section{Type and Token Ratio}

La literatura científica ha registrado una correlación entre una variedad léxica restringida y la predicción de una eventual discapacidad cognitiva desde la infancia. En el caso de la esquizofrenia, nos encontramos con una disminución tanto en la variedad como en el volumen léxico, y además, con el empleo de palabras de escasa frecuencia de aparición o palabras rebuscadas, aplicadas a contextos semánticos no pertinentes. Entre los grupos de esquizofrenia, encontramos en G1-CRE un volumen léxico inferior al grupo de los controles y superior al G2-PEE. Llama la atención que esta tendencia se mantuvo en la medición de todos los indicadores estudiados aquí. Entre el grupo G1-CRE se observó una mayor proporción de ítems léxicos de baja frecuencia de aparición en relación con los grupos G2-PEE y G3-CONT. Cabe destacar que los tres escritores con TFP pertenecían al grupo G1-CRE y que su desempeño fue concordante con el resto de los escritores con patología.

El control inhibitorio, que se encuentra activo en la fase del primer episodio, puede ser responsable de las diferencias de volumen léxico con los hablantes crónicos. Además, los factores demográfico-clínicos son menos favorables para los escritores de primer episodio, debido a que por su edad y la aparición de la psicosis, cuentan con una formación académica incompleta.

\section{Longitud de las palabras}

Los resultados obtenidos en esta medición confirman lo que ha señalado la literatura respecto de la preferencia de palabras bisilábicas, en el caso del grupo control. No obstante, tanto el grupo G1-CRE y G2PEE presentan, en promedio, palabras simples que no logran componerse por dos sílabas. Por tanto, es posible determinar que existe una 
predominancia por palabras monosilábicas que, en el español, son en su mayoría palabras gramaticales. Es decir, se aprecia una pobreza léxica en los grupos con diagnóstico de esquizofrenia. Además, en concordancia con los demás resultados, también encontramos el uso de ítems léxicos rebuscados, que no aportan contenido relevante.

\section{Longitud de las oraciones}

La simplificación oracional está asociada al deterioro cognitivo, donde la memoria de trabajo se hace menos eficiente y, por ende, se producen oraciones más breves. Pensamos que con el uso predominante de ítems léxicos breves también se elaboran oraciones breves y se produce una inevitable simplificación sintáctica.

A pesar de que en longitud de las oraciones los resultados demuestran que el grupo de G1-CRE obtiene un levemente mayor número de palabras por oraciones que los controles, lo que varía es la complejidad sintáctica (hay una menor cantidad de estructuras complejas gramaticalmente). Esto se observará en un trabajo posterior que se encuentra en preparación. Aunque este valor no es significativo estadísticamente entre controles y G2; entre G1 y G2 sí hay una diferencia significativa importante.

\section{Categorías gramaticales}

En este indicador se mantuvo la tendencia de rendimiento de los tres grupos en los indicadores anteriores. Se destaca la diferencia en la frecuencia de uso de las palabras de categoría gramatical, en comparación con aquellas palabras referenciales. Por ejemplo, los tres grupos en estudio destacan una preferencia por los sustantivos, verbos y adjetivos. Por el contrario, son los G3-CT quienes presentan un mayor uso de palabras gramaticales. Esto es interesante, pues en la medición de longitud de las palabras, sugerimos que en G1-CRE y G2-PEE existe una predominancia por palabras monosilábicas, ya que no logran superar las dos sílabas por palabra, a diferencia del grupo control. Esto sugeriría una pobreza léxica, pues coincidiría con un mayor uso de palabras cortas como las preposiciones, artículos o conjunciones.

Sería adecuado proponer que el análisis de la longitud de las oraciones y el TTR podría explicar esta distinción. Es decir, en proporción, los grupos G1-CRE y G2-PEE presentan un menor volumen léxico y 
longitud de oraciones. En este sentido, sería pertinente profundizar estos resultados, considerando cuál es la función gramatical de las palabras dentro de la construcción de las oraciones y evaluar si existe una escritura coherente. Por tanto, en este estudio presentamos un análisis cuantitativo respecto del uso de palabras, con el fin de evidenciar una pobreza léxica en los grupos diagnosticados con esquizofrenia.

\section{Conclusión}

Frente a una tarea de elaboración libre es necesaria la articulación de diferentes procesos cognitivos que facilitan evocación de ideas, la planificación y la toma de decisiones para establecer objetivos dentro del texto. La demanda cognitiva pone a prueba las capacidades de organización conceptual para elaborar representaciones de mundo. Sumada a esta demanda, la organización textual requiere un automonitoreo constante durante el transcurso de la tarea, a fin de asegurar un producto final. Teniendo en cuenta que las competencias de escritura se aprenden en la enseñanza primaria, la escritura creativa de un texto breve no representa un desafío excesivo.

Al comparar los textos entre los grupos de este estudio podemos verificar que, por una parte, las diferencias apuntan a que en los grupos de esquizofrenia existe una importante disminución en las habilidades de automonitoreo. Esta disminución incide en la producción de textos con menor volumen léxico y complejidad oracional. Por otra parte, las representaciones de mundo están centradas en descripciones informativas de poco vuelo creativo, lo que es una posible consecuencia del deterioro en el lóbulo frontotemporal, que es la región cerebral que se encarga de los aspectos creativos que, en buenas cuentas, son habilidades que contribuyen a un mejor desempeño social. Asimismo, la escritura se relaciona con procesos de razonamiento y pensamiento creativo que presentan un complejo equilibrio (Abraham et al., 2005; Schmidt y Seger, 2009).

Una de las limitaciones del estudio se centra en el tamaño de la muestra acotada que se analizó. Resulta primordial configurar un registro de mayor volumen que facilite la caracterización léxica en tareas de escritura. Pese a esta dificultad, los resultados obtenidos en este trabajo sugieren que es posible proponer una caracterización parametrizable de rasgos diferenciales en la escritura de personas con 
diagnóstico de esquizofrenia. A nivel teórico, los resultados parecen proporcionar evidencia en relación con algunas hipótesis en la investigación de la esquizofrenia, como por ejemplo la propuesta de quienes subrayan la enorme importancia del marco lingüístico del pensamiento en la esquizofrenia para explicar los fenómenos de desviación en el discurso. Ellos se basan en la hipótesis de que la desorganización lingüística en el cerebro afectado por la psicosis desempeña un papel central en la comprensión de la patogénesis de la enfermedad, por tanto, sería uno de los posibles rasgos explicativos de formas de cognición asociadas a la patología. A este respecto, en este breve análisis, hemos encontrado evidencias a favor de la necesidad de elaborar modelos explicativos que puedan aplicarse a diferentes aspectos del lenguaje esquizofrénico, en el marco estricto del trabajo clínico y terapéutico.

Una de las proyecciones de este estudio puede ser el diseño de un instrumento que incluya, además de los indicadores clínicos, un marco para evaluar el desempeño en escritura. A fin de cautelar los posibles sesgos que generan las evaluaciones psicopatológicas, sería deseable que la elaboración clínica de mediciones del desempeño léxico se configuren como un instrumento diseñado específicamente para esquizofrenia o para cuadros o estados psicóticos.

\section{Referencias bibliográficas}

Abraham, A., Windmann, S., Daum, I., y Güntürkün, O. (2005). Conceptual expansion and creative imagery as a function of psychoticism. Consciousness and Cognition, 14(3), 520-534. https://doi.org/10.1016/j.concog.2004.12.003

Addington, J. y Addington, D. (2000). Neurocognition in first episode schizophrenia: A 2 year follow-up. Schizophrenia Research, 41(1), 271-272. https://doi.org/10.1016/s09209964(00)90984-8

Allen, H. A., Liddle, P. F., y Frith, C. D. (1993). Negative features, retrieval processes and verbal fluency in schizophrenia. British Journal of Psychiatry, 163(6), 769-775. https://doi.org/10.1192/ bjp.163.6.769

Altemeier, L., Jones, J., Abbott, R. D., y Berninger, V. W. (2006). Executive functions in becoming writing readers and reading wri- 
ters: Note taking and report writing in third and fifth graders. Developmental Neuropsychology, 29(1), 161-173. https://doi. org/10.1207/s15326942dn2901_8

Andreasen, N. C. y Grove, W. M. (1986). Thought, language, and communication in schizophrenia: Diagnosis and prognosis. Schizophrenia Bulletin, 12(3), 348-359. https://doi.org/10.1093/ schbul/12.3.348

Baddeley, A. (2003). Working memory and language: An overview. Journal of Communication Disorders, 36(3), 189-208. https:// doi.org/10.1016/s0021-9924(03)00019-4

Bardin, L. (1991). Análisis de contenido. Ediciones AKAL.

Benson, D. F. y Stuss, D. T. (1990). Frontal lobe influences on delusions: A clinical perspective. Schizophrenia Bulletin, 16(3), 403411. https://doi.org/10.1093/schbul/16.3.403

Berninger, V. W. y Winn, W. D. (2006). Implications of advancements in brain research and technology for writing development, writing instruction and educational evolution. C. A. MacArthur, S. Graham, y J. Fitzgerald (Eds.), Handbook of writing research (pp. 96-114). The Guilford Press.

Castilla del Pino, C. y Ruíz-Vargas J. M. (Coords.) (1991). Aspectos cognitivos de la esquizofrenia. Trotta.

Carlino, P. (2009). Escribir, leer y aprender en la universidad: una introducción a la alfabetización académica. Fondo de Cultura Económica.

Carreras-Riudavets, F., Santana-Herrera, J. C., Hernández-Figueroa, Z., Rodríguez-Rodríguez, G. (2011). Parametrizador morfológico de textos-ParamText TIP. http://tulengua.es

Cassany, D. (2002). La cocina de la escritura. Anagrama.

Crow, T. J. (1997). Is schizophrenia the price that Homo sapiens pays for language? Schizophrenia Research, 28(s 2-3), 127-141. https://doi.org/10.1016/s0920-9964(97)00110-2

Cuetos, F. (2012). Neurociencia del lenguaje: bases neurológicas e implicaciones clínicas. Editorial Médica Panamericana.

Datta, D. y Arnsten, A. F. T. (2018). Unique molecular regulation of higher-order prefrontal cortical circuits: Insights into the Neurobiology of Schizophrenia. ACS Chemical Neuroscience, 9(9), 2127-2145. https://dx.doi.org/10.1021\%2Facschem neuro.7b00505 
DSM-IV (1997) Manual Diagnóstico y Estadístico de los Trastornos Mentales. American Psychiatric Association.

Escandell, M. V. (Coord.) (2014). Claves del lenguaje humano. Editorial Universitaria Ramón Areces.

Figueroa Barra, A. (2015). Análisis pragmalingüístico de los marcadores de coherencia en el discurso de sujetos con esquizofrenia crónica y de primer episodio [Tesis doctoral]. Universidad de Valladolid.

Figueroa, A., Durán, E., y Oyarzún, S. (2017). La gestión temática como marcador de déficit lingüístico primario en personas con diagnóstico de primer episodio de esquizofrenia: un estudio en una muestra chilena. RLA. Revista de Lingüística Teórica y Aplicada, 55(1), 117-147. https://doi.org/10.4067/s071848832017000100117

Figueroa, A (en prensa). La referencialidad en la psicosis. En P. LópezSilva (Ed.), La realidad transformada: ensayos en psicopatología contemporánea. Ediciones Universidad de Valparaíso.

Figueroa, A., Oyarzún, S., y Durán, E. (2019). CONECTA-2: entrenamiento de la cognición social y el lenguaje, al servicio de la eficacia comunicativa. Una experiencia clínica interactiva con personas con diagnóstico de esquizofrenia. Revista Chilena de Neuro-Psiquiatría, 57(1), 9-18. https://doi. org/10.4067/s0717-92272019000100009

First, M. B., Spitzer, R. L., Gibbon, M., \& Williams, J. B. W. (2002). Structured clinical interview for DSM-IV-TR axis I disorders, research version, patient edition. (SCID-I/P). New York: Biometrics Research, New York State Psychiatric Institute.

Fletcher, P. (1985) A child's learning of English. Blackwell.

Hayes, J. y Flower, L. (1980). Identifying the organization of writing processes. Cognitive processes in writing. En L. Gregg y E. Teinberg (Eds.), Cognitive processes in writing: An interdisciplinary approach (pp. 3-30). Erlbaum.

Hayes, J. R. y Flower, L. S. (1990). Expert planning processes in writing. Final report. https://doi.org/10.21236/ada231380

Hinzen, W. y Sheehan, M. (2013). The philosophy of universal grammar. Oxford University Press.

Kay, SR, Opler LA, Spitzer, RL, Williams JB, Fiszbein and Gorelick, A.(1991)SCID-PANSS: two-tier diagnostic system for psychotic disorders. 1991. Compr Psychiatry. Jul-Aug;32(4):355-61. 
Kirk, S. A. (1951). The development of reasoning in children with normal and defective hearing. Mildred C. Templin. The Elementary School Journal, 51(9), 533-534. https://doi. org/10.1086/459291

Kircher, T., Krug, A., Stratmann, M., Ghazi, S., Schales, C., Frauenheim, M., Turner, L., Fährmann, P., Hornig, T., Katzev, M., Grosvald, M., Müller-Isberner, R., y Nagels, A. (2014). A rating scale for the assessment of objective and subjective formal Thought and Language Disorder (TALD). Schizophr. Res., 160(1-3), 216-221. https://doi.org/10.1016/j.schres.2014.10.024

Liederman, J., Kohn, S., Wolf, M., y Goodglass, H. (1983). Lexical creativity during instances of word-finding difficulty: Broca's vs. Wernicke's aphasia. Brain and Language, 20(1), 21-32. https://doi.org/10.1016/0093-934x(83)90029-9

Malvern, D., Richards, B., Chipere, N., y Durán, P. (2004). Traditional approaches to measuring lexical diversity. En D. Malvern, B. Richards, N. Chipere, y P. Durán, P (Eds.), Lexical Diversity and Language Development (pp. 16-30). Palgrave Macmillan. https://doi.org/10.1057/9780230511804_2

McKenna, P. J. y Oh, T. M. (2005). Schizophrenic speech: Making sense of bathroots and ponds that fall in doorways. Cambridge University Press.

Miller, J. F. (1981). Assessing language production in children: Experimental procedures. Edward Arnold.

Miller, J. F. y Chapman, R. S. (1981). The relation between age and mean length of utterance in morphemes. Journal of Speech and Hearing Research, 24(2), 154-161. https://doi.org/10.1044/ jshr.2402.154

Morice, R. D. e Ingram, J. C. L. (1983). Language complexity and age of onset of schizophrenia. Psychiatry Research, 9(3), 233-242. https://doi.org/10.1016/0165-1781(83)90048-3

Nuechterlein, K. H. y Dawson, M. E. (1984). A heuristic vulnerability/ stress model of schizophrenic episodes. Schizophrenia Bulletin, 10(2), 300-312. https://doi.org/10.1093/schbul/10.2.300

Okocha, I. y Toone, B. (1992). Frontal lobe impairment in schizophrenia. Schizophrenia Research, 6(2), 158-159. https://doi. org/10.1016/0920-9964(92)90245-z

Olson, D. (1998). El mundo sobre el papel. El impacto de la escritura y la lectura en la estructura del conocimiento. Gedisa. 
Ong, W. J. (1987). Oralität und Literalität. Die Technologisierung des Wortes. Springer. https://doi.org/10.1007/978-3-322-910677

Pittman-Polletta, B. R., Kocsis, B., Vijayan, S., Whittington, M. A., y Kopell, N. J. (2015). Brain rhythms connect impaired inhibition to altered cognition in schizophrenia. Biological Psychiatry, 77(12), 1020-1030. https://doi.org/10.1016/j.biopsych.2015.02.005

Potgieser, A. R. E., van der Hoorn, A., y de Jong, B. M. (2015). Cerebral activations related to writing and drawing with each hand. PloS One, 10(5), e0126723. https://doi.org/10.1371/journal. pone. 0126723

Ramón, P. R., Arias-Gundín, O., Palomo, M. P., Andina, E., y Rodríguez, C. (2018). Perfil escritor y conocimiento metacognitivo de las tareas académicas en los estudiantes universitarios. $\mathrm{Pu}$ blicaciones, 48(1). https://doi.org/10.30827/publicaciones. v48il.7335

Real Academia Española (s.f.). Corpus de referencia del español actual, CREA. Recuperado en 12 de juniode 2020, de http://corpus. rae.es/lfrecuencias.html

Retherford, R. D. y Choe, M. K. (1993). Statistical models for causal analysis https://doi.org/10.1002/9781118033135

Separar en sílabas (2018). Separar en sílabas. Separador y contador de sílabas, analizador de poesía y textos. https://www.separarensilabas.com/index.php

Schmidt, G. L. y Seger, C. A. (2009). Neural correlates of metaphor processing: The roles of figurativeness, familiarity and difficulty. Brain and Cognition, 71(3), 375-386. https://doi. org/10.1016/j.bandc.2009.06.001

Van Dijk, T. A. y Kintsch, W. (1978). Toward a model of text comprehension and production. Psychological Review, 85(5), 363-394. https://psycnet.apa.org/doi/10.1037/0033-295X.85.5.363

Zimmerer, V., Watson, S., Turkington, D., Ferrier, N., e Hinzen, W. (2017). Deictic and propositional meaning-new perspectives on language in schizophrenia. Front Psychiatry, 8(17). https://doi.org/10.3389/fpsyt.2017.00017

Zipf, G. K. (1972). Human behaviour and the principle of least effort. An introduction to human ecology. Addison-Wesley. Trabajo publicado originalmente en 1949. 